\title{
MODELING APPROACH AND FINITE ELEMENT ANALYSES OF A SHAPE MEMORY EPOXY-BASED MATERIAL
}

\author{
Davide Battini ${ }^{1}$, Andrea Avanzini ${ }^{1}$, Stefano Pandini ${ }^{1}$, Fabio Bignotti ${ }^{1}$ \\ ${ }^{1}$ Department of Mechanical and Industrial Engineering, University of Brescia \\ via Branze 38, 25123, Brescia, Italy \\ E-mail: davide.battini@unibs.it, andrea.avanzini@unibs.it,stefano.pandini@unibs.it, \\ fabio.bignotti@unibs.it
}

Keywords: shape memory polymer, thermo-mechanical model, finite element, epoxy resin, DMA, viscoelasticity

\begin{abstract}
A series of structurally related epoxy resins were prepared as model systems for the investigation of the shape memory response, tailoring their thermo-mechanical response and describing their strain evolution under triggering stimuli with a thermo-viscoelastic model. The shape memory behavior on epoxy resin was modeled through the definition of linear viscoelastic parameters, in combination with the general time-temperature reduction scheme. Specifically, this translates into the definition of a hyperelastic response enriched with a Prony series to implement time dependency and a William-Landel-Ferry (WLF) equation to implement temperature dependency. While the hyperelastic response parameters are found with a standard fitting procedure on compression tests, finding the correct parameters for the Prony series might be challenging. For this reason, an ad-hoc optimization process was coded in Mathworks Matlab environment: proper guess values are created and then a chain of constrained optimizers (such as genetic, particle swarm, pattern search and different nonlinear programming algorithms) with smart evolving boundaries looks for the right set of parameters. The ability to correctly predict strain history and shape transitions with a finite element model was evaluated on a case study for self-deployment of a folded tubular structure. Tubular specimens were tested and the model was used to reproduce the switching from a temporary folded six-pointed star shape to their original cylindrical shape. Overall, this approach proved to be a very effective way to simulate complex shape memory responses in time and temperature domains, for which standard Dynamic Mechanical Analyses (DMA) and uniaxial tensile or compression tests are sufficient to calibrate material parameters for Finite Element (FE) implementation.
\end{abstract}




\section{INTRODUCTION}

Shape memory (SM) polymers (SMP) are stimuli-responsive materials with the ability of changing shape upon an external trigger. In fact, SMP can be considered part of the family of the smart materials since they can 'sense' the environment and change their shape accordingly. In general, SMP can undergo reversible large deformations and their deformed temporary shape can be fixed by means of a proper thermal, electrical, chemical or mechanical load history until an external stimulus trigger the recovery of the original shape undeformed shape. Shape recovery of SMP can be defined as one-way if they can only switch from the temporary shape to the original shape once or as two-way if they can switch back and forth from one shape to the other. SMP can also exhibit from one (dual-shape) up to several (multi-shape) stable temporary shape $[1,2]$.

Thermal stimuli are the most common methods used for activating SMP and forcing a switch of their shape. Thermally-stimulated transformations are usually linked with the glass transition temperature $\left(\mathrm{T}_{\mathrm{g}}\right)$ or the melting temperature $\left(\mathrm{T}_{\mathrm{m}}\right)$ of the polymer [3, 4]. Chemically crosslinked polymers allow the control of their switching temperature and thermo-mechanical behavior with proper tailoring of their network structure. Thus, the choice of epoxy resins can be ideal since a proper selection of their formulation (resin/hardener type and ratio, addition of modifiers) enables a good control of their molecular architecture with relative ease [5-9].

Due to their interesting behavior, SMP and their composites are appealing for many innovative devices targeted towards different fields: biomedical, aerospace, construction engineering, mechanical, purely aesthetic, etc. [3, 8, 10]. Moreover, with the ongoing rise of 3D printing, the interest in (SMP) for engineering applications has grown even higher. However, the lack of effective approaches to include the modelling of shape memory effects in the design process of a real component could hinder the potential advantages associated with an easier and cheaper production of such smart devices. In this sense, the employment of linear viscoelastic parameters in combination with the general time-temperature reduction scheme was shown to be effective in recent studies [11-15]. Moreover, this approach is very practical due to its implementation in well-known commercial finite element method (FEM) software such as DS Simulia Abaqus and due to the requirement of feasible experimental testing such as static uniaxial tests and multifrequency Dynamic Mechanical Analysis (DMA) or stress relaxation tests at various temperatures (to provide full description of the material properties in terms of master curves).

Specifically, this translates into the definition of a hyperelastic response enriched with a Prony series to implement time dependency and a William-Landel-Ferry (WLF) equation to implement temperature dependency. While the hyperelastic response parameters are found with a standard fitting procedure on uniaxial tests, finding the correct parameters for the Prony series might be challenging. For this reason, an ad-hoc optimization process was coded in Mathworks Matlab environment: proper guess values are created and then a chain of constrained optimizers (such as genetic, particle swarm, pattern search and different non-linear programming algorithms) with smart evolving boundaries looks for the right set of parameters.

In this work the practical effectiveness of this modeling approach was assessed by its application to the shape memory behavior of a previously tailored epoxy resin [5] which was characterized via feasible and "common" experimental tests [5]. A just as feasible finite element material modelling approach is then validated via star-shaped recovery experimental tests.

\section{MATERIALS AND METHODS}

An epoxy resin system was prepared staring from resin DGEBA 355, 4-40 diaminodiphenylmethane and phenyl glycidyl ether (PGE), that were purchased from Sigma- 
Aldrich Co. (Saint Louis, USA) and used without purification. The epoxy resin was prepared with a specific formulation, identified through the stoichiometric ratio, q, and the PGE fraction, fPGE. These parameters, whose definition may be found in a companion paper [16], and for this specific system were chosen as follows $\mathrm{q}=0.96$; $\mathrm{fPGE}=0.45$, as, under this formulation, it was possible to achieve a ductile response above $\mathrm{T}_{\mathrm{g}}$ (about $35^{\circ} \mathrm{C}$ ), allowing to greatly deform the specimen with no risk of brittle failure. Specimens were prepared by casting the mixed resin in silicon molds, according to three geometries:

- Rectangular specimens (length $=90 \mathrm{~mm}$; width $=7 \mathrm{~mm}$; thickness $=1 \mathrm{~mm}$ ), employed for the DMA characterization.

- Cylindrical specimens (height $=10 \mathrm{~mm}$; diameter $=7.5 \mathrm{~mm}$ ), employed for the mechanical characterization above $\mathrm{T}_{\mathrm{g}}$.

- Tubular specimens (height $=30 \mathrm{~mm}$; external diameter $=26 \mathrm{~mm}$; thickness $=0.5$ and $1.5 \mathrm{~mm}$ ), employed for the shape memory testing.

More details concerning resin and specimens preparation can be found in [16].

\subsection{Experimental characterization}

The epoxy resin was experimentally characterized via uniaxial compression tests and Dynamic Mechanical Analysis (DMA). Uniaxial compression tests aimed at gathering data on the behavior of the material in its rubbery state and were performed by means of an electromechanical dynamometer (Instron Mod. 3366) on cylindrical specimens, whose faces were abraded with sandpaper to ensure adequately flat and parallel faces at the extremities. The compression plate surfaces were lubricated with paraffin oil before each test in order to reduce the friction between the specimens and the plates. The tests were carried out at $85^{\circ} \mathrm{C}$ under displacement control, at a fixed displacement rate of $0.5 \mathrm{~mm} / \mathrm{min}$.

On the other hand, DMA tests aimed at gathering data on the viscoelastic behavior of the epoxy resin thus allowing the definition of a time-temperature correlation. DMA tests were carried out on rectangular bars under tensile configuration, by means of a DMA apparatus (DMA Q800 - TA Instruments), operating in frequency sweeps from 3 to $100 \mathrm{~Hz}$, with a $10 \mu \mathrm{m}$ amplitude and by heating at $0.50{ }^{\circ} \mathrm{C} / \mathrm{min}$ from room temperature to $100^{\circ} \mathrm{C}$. Starting from each frequency sweep, a time-temperature reduction scheme was then employed to obtain the storage modulus vs reduced frequency master curve and the correlation between shift factors and temperature.

\subsection{Material modelling}

We opted for a visco-hyperelastic modelling where a proper hyperelastic strain energy function is chosen to fit the non-linear static (fully relaxed and thus time-independent) behavior of the epoxy resin and then enriched with a generalized Maxwell model to introduce time dependency and a William-Landel-Ferry (WLF) equation to introduce a time-temperature correlation [5, 11-15]. The shift factors $a_{T}$ allow switching from the temperature to the time domain with a simple scale factor: i.e. considering a fixed frequency $\omega_{0}$, a change from the temperature $T_{0}$ to $T$ can be regarded as a change in frequency from $\omega_{0}$ to $a_{T, 0} \omega_{0}$ with the temperature fixed at $T_{0}$. This theoretical framework $[17,18]$ leads to the definition of a shift factor function $a_{T}(T)$ and in our case, the WLF equation, see eq. (1), is introduced to fit the $a_{T}(T)$ that were calculated to obtain the master curve from multi-frequency DMA data:

$$
\log \left(a_{T}\right)=\frac{-C_{1}\left(T-T_{0}\right)}{C_{2}+\left(T-T_{0}\right)} \quad \text { with } \quad f_{R}=a_{T} f \quad \text { and } \quad \omega=2 \pi f
$$

where $C_{1}$ is a dimensionless coefficient, $C_{2}$ is a coefficient with dimensions of temperature, 
$T_{0}$ is the reference temperature, $T$ is the temperature associated with the shift factor $a_{T}$ and $f_{R}$ and $f$ are, respectively, the reduced frequency and the DMA data frequency. Data fitting for $C_{1}$ and $C_{2}$ is straightforward and was carried out with standard nonlinear least squares method (starting from reasonable guess values). On the other hand, the generalized Maxwell model [19] allows describing the relaxation function of the complex dynamic modulus $G^{*}$ through its components storage $\left(G^{\prime}\right)$ and loss components $\left(G^{\prime \prime}\right)$ as $G^{*}=G^{\prime}+i G^{\prime \prime}$. Considering the DMA apparatus, the specimen undergoes a cyclic loading and the phase shift between the stress and strain response is usually referred as $\delta$. This leads to the definition of a dissipation factor $\tan (\delta)=G^{\prime \prime} / G^{\prime}$ that tends to zero for an elastic material (no stress-strain phase shift) and to infinite for a viscous material ( $\pi / 2$ stress-strain phase shift) $[17,18]$.

In our case a Prony series [20] is introduced to describe the generalized Maxwell model and couple the linear viscoelasticity theoretical framework with the hyperelastic static response. Therefore, a relaxation function can be expressed for the storage, $G^{\prime}(\omega)$, and the loss, $G^{\prime \prime}(\omega)$, shear moduli as per eq. (2) and (3) respectively:

$$
\begin{gathered}
G^{\prime}(\omega)=G_{0}\left(1-\sum_{i=1}^{N} g_{i}+\sum_{i=1}^{N} \frac{g_{i} \tau_{i}^{2} \omega^{2}}{1+\tau_{i}^{2} \omega^{2}}\right) \\
G^{\prime \prime}(\omega)=G_{0} \sum_{i=1}^{N} \frac{g_{i} \tau_{i} \omega}{1+\tau_{i}^{2} \omega^{2}}
\end{gathered}
$$

where $G_{0}$ is the instantaneous shear modulus, $\omega$ is the angular reduced frequency and $\left(g_{i}, \tau_{i}\right)$ are the coefficients of the Prony series with $g_{i}$ being dimensionless and $\tau_{i}$ having the dimensions of $\omega^{-1}$. Since $G^{\prime}=E^{\prime} / 3$ and $G^{\prime \prime}=E^{\prime \prime} / 3$, DMA data can be used to find optimal $\left(g_{i}, \tau_{i}\right)$ values, but fitting the Prony series is way less trivial than fitting the WLF equation and thus a proper optimization routine was coded in Mathworks Matlab 9.5 (2018) environment. In fact, the Prony series might require a high number of coefficients (even over 25 pairs) for an optimal fit and the error function describing the total residual between the analytical forms expressed as per eq. (2) and (3) and experimental data shows many local minima. Moreover, it is very important for the optimization routine to include proper boundaries for the $\tau_{i}$ to span over the whole experimental data range and proper constraints for the $g_{i}$ to fulfil that:

$$
\sum_{i=1}^{N} g_{i}<1 \quad \text { since } G_{\infty}=G_{0}\left(1-\sum_{i=1}^{N} g_{i}\right)
$$

with $G_{\infty}$ being the fully relaxed shear modulus.

The optimization routine is provided with a Graphical User Interface (GUI) where the user can tweak some basic settings like choosing the experimental data file to fit, the number of Prony coefficients, the solvers, the number of iterations, etc. Overall, the optimization routine follows the procedure shown in Figure 1 in order to achieve consistent results and a robust fitting. 


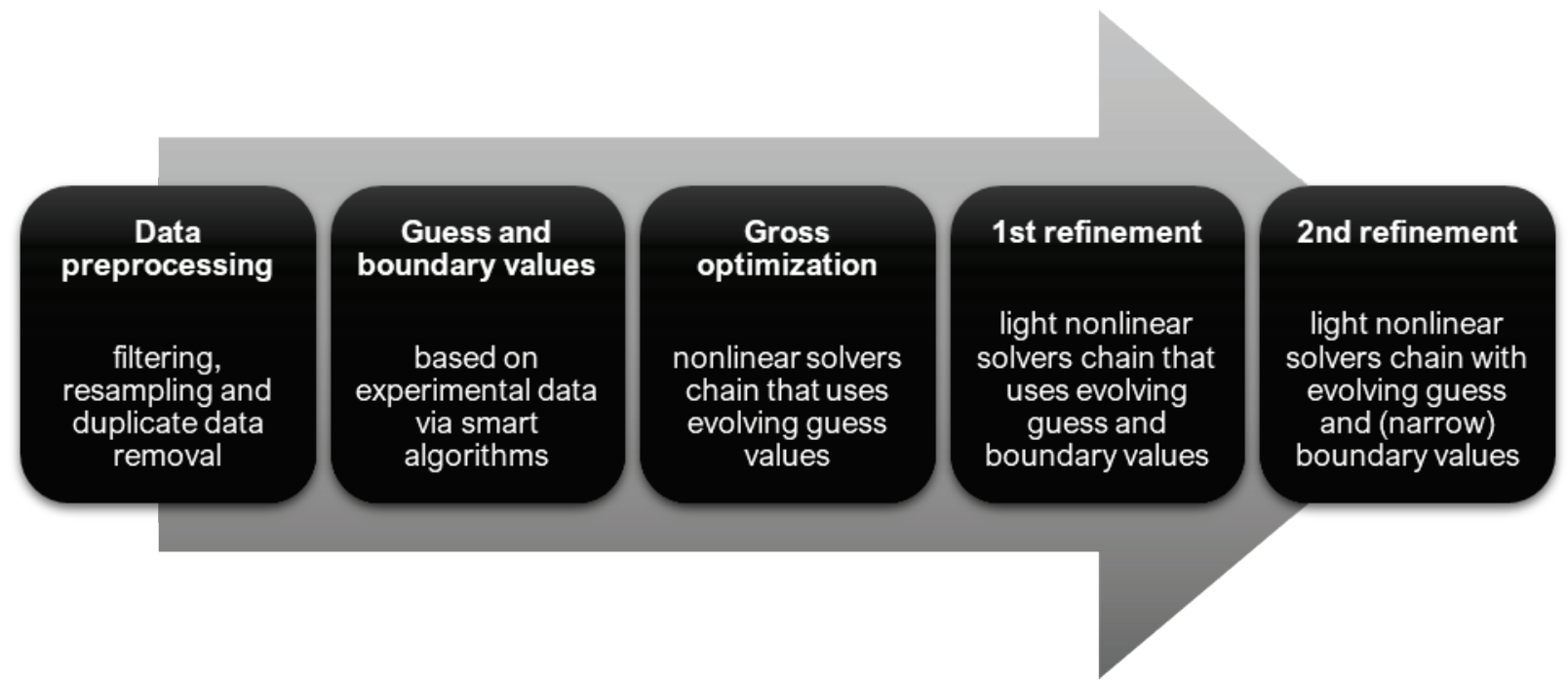

Figure 1: Optimization procedure used to fit Prony series parameters on the multi-frequency master curve obtained from DMA data.

With reference to Figure 1, the code can be described through its five phases:

1) The software automatically removes duplicate data and calculates data weights to account for any removed data point. It also offers the possibility of preprocessing DMA data with filters such as high-pass, low-pass or data smoothing, and many resampling rules (linear, logarithmic or even biased). Data smoothing is provided through a very classic but effective triangular (1-2-1) filter which the user can apply $\mathrm{N}$ times [21, 22]. Both proper filtering and resampling of input data can result in better convergence rate and an overall better solution of the optimization routine.

2) Once experimental data are fully preprocessed, proper guess and boundary values for both $\tau_{i}$ and $g_{i}$ are either calculated automatically or fixed manually. In case of the automatic calculation, the algorithm uses a logarithmic spacing for the $\tau_{i}$ and covers the whole reduced frequency range of the experimental data while the $g_{i}$ distribution is guessed from the storage modulus curve. In terms of boundaries, while the $g_{i}$ have uniform limits set at a suggested user value, different algorithms are coded for the $\tau_{i}$, with the automatic one setting the lower and upper boundaries depending on the $\tau_{i}$ distribution in order to limit the possible overlapping (during optimization) between $\tau_{i-1}, \tau_{i}$ and $\tau_{i+1}$ boundaries.

3) At this point, the optimization routine starts with a first gross optimization that can chain up to 5 different solvers (genetic algorithms, pattern search, nonlinear programming solvers and particle swarm). The solver choice was determined by taking into consideration feasibility (most of these solver codes are already implemented and ready-to-use) and solver typology (i.e. a mixture of evolutionary, gradient-based, derivative-free, direct search, etc.) $[23,24]$ and ease of constraint and boundary implementation. Specifically, every solver of this chain is constrained to eq. (4) and uses the same boundary values but only the first one uses the guess values calculated above since each subsequent solver will use the optimized output of the previous solver as new guess values. Chaining different solvers usually improves the overall optimization by lowering the chance of getting stuck into a bad local minimum. The user can also customize the chain order or even disable any of the solvers to double check to check the goodness of the optimized solution (without the need of perturbing the guess values via trial and error approach). Furthermore, all the solvers are set by default with appropriate options in order to be more robust against bad local minima by sacrificing a little the convergence rate 
and/or the processing time: for example, the genetic algorithm uses crossover ratios lower than usual values and the pattern search uses complete search/polling.

4) After the first chain optimization two refinement chain optimizations can also be performed. The first refinement chain works similarly to the roughing chain but with two differences: it relies on pattern search and nonlinear programming solvers only (due to their speed and convergence rate) and evolves boundaries on solver switch by applying a modified version of the previously described automatic boundaries calculator. This step usually helps in getting a better solution but still leaves some room for abrupt changes in the optimized $\tau_{i}, g_{i}$.

5) The second and last refinement chain is almost identical to the previous one with the only difference of having a boundary evolution algorithm that provides way narrower limits to guarantee only small changes in the solution.

In the end an optimized solution is available and plots of $G^{\prime}, G^{\prime \prime}$ and $\tan (\delta)$ are shown to the user to quickly compare experimental data with the guessed and optimized solutions. If needed, the user is also provided some secondary settings to tweak the error function by modifying the weights of input data and switching between linear and logarithmic error calculations.

\subsection{Star folding tests}

The shape memory response of the material was evaluated on the tubular specimens, by first folding them into a six-arms star-shaped temporary configuration, possibly resembling some stent prototypes [25], and later monitoring the recovery of their original shape. The shape memory effect was studied as a function of time and temperature by measuring the timeevolution of the specimen shape during isothermal recovery tests (similarly to $[26,27]$ ) with all the testing conditions being summarized in Table 1.

\begin{tabular}{|c|c|c|c|c|}
\hline Specimen \# & $\begin{array}{c}\text { External } \\
\text { diameter } \\
{[\mathrm{mm}]}\end{array}$ & $\begin{array}{c}\text { Thickness } \\
{[\mathrm{mm}]}\end{array}$ & $\begin{array}{c}\text { Punch radial } \\
\text { displacement } \\
{[\mathrm{mm}]}\end{array}$ & $\begin{array}{c}\text { Recovery } \\
\text { temperature } \\
{\left[{ }^{\circ} \mathrm{C}\right]}\end{array}$ \\
\hline Thick 1 & 26 & 1.5 & 3.2 & 37 \\
\hline Thick 2 & 26 & 1.5 & 5.6 & 38.5 \\
\hline Thick 3 & 26 & 1.5 & 5.4 & 42 \\
\hline Thick 4 & 26 & 1.5 & 3.2 & 43 \\
\hline Thin 1 & 26 & 0.5 & 4.3 & 37 \\
\hline Thin 2 & 26 & 0.5 & 8.9 & 38.5 \\
\hline Thin 3 & 26 & 0.5 & 8.2 & 42 \\
\hline Thin 4 & 26 & 0.5 & 5.8 & 43 \\
\hline
\end{tabular}

Table 1: Parameters describing the testing condition of the 8 samples.
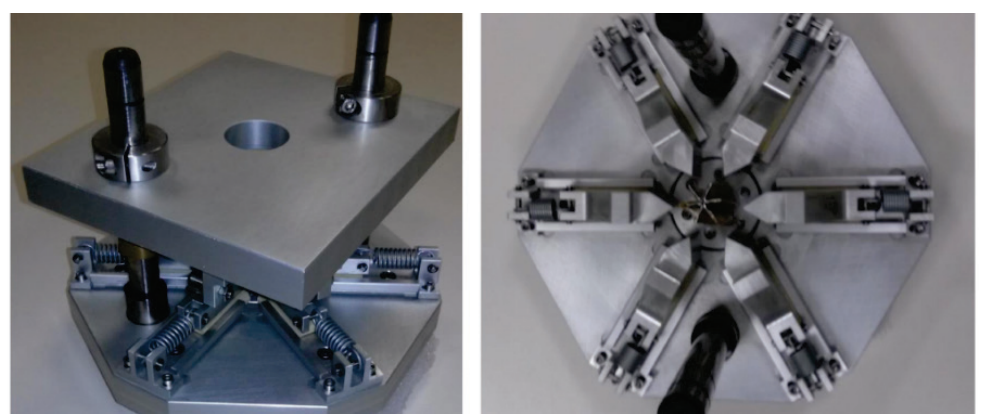

Figure 2: Device used for the programming phase of the tubular specimen into the six-pointed star geometry. 
The tubular specimens were heated at about $80{ }^{\circ} \mathrm{C}$ and crimped in a star configuration by means of an ad-hoc designed tool (see Figure 2); to effectively fix this temporary configuration, the deformed specimen, within the crimping tool, were put in a refrigerator at $-17{ }^{\circ} \mathrm{C}$ and maintained at this temperature prior than testing. The shape memory effect was promoted by putting the specimen in an oven at a given temperature (above $\mathrm{T}_{\mathrm{g}}$ ) in order to allow a recover under stress-free conditions. The shape change was tracked by means of a photo-camera, at a sampling frequency of 1 frame/s. A qualitative representation of the temperature and strain histories of the specimen is given in Figure 3.

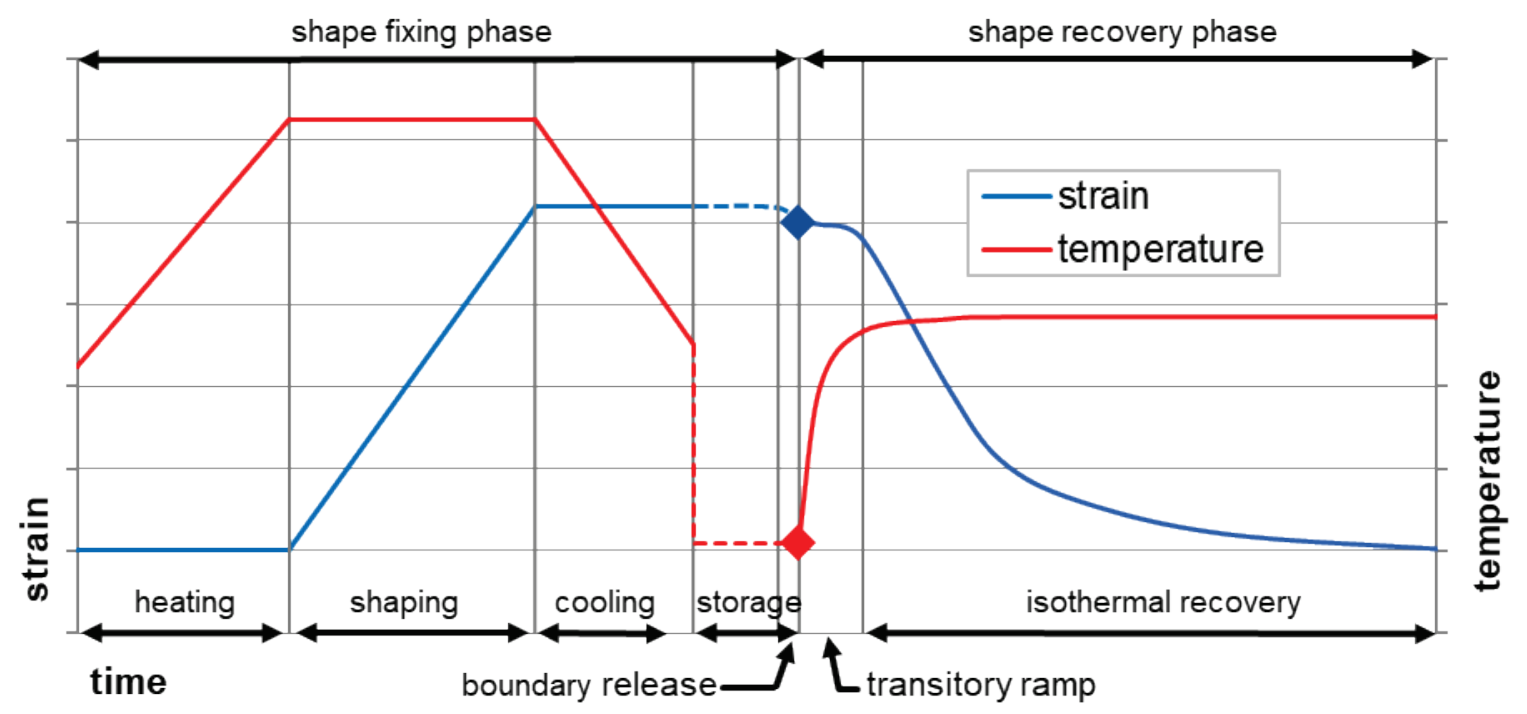

Figure 3: Qualitative temperature and strain histories of the shape fixing and shape recovery phases.

The recovery process was described in terms of an arbitrary parameter based on the section of the folded specimen. We refer to this parameter as the normalized deformation factor, $\varphi_{n}$, calculated in eq. (5):

$$
A_{0}=\pi R_{i, 0}^{2} \quad \Rightarrow \quad \varphi_{n}(t)=\frac{A_{0}-A_{\text {star }}(t)}{A_{0}-A_{\text {star }, 0}}
$$

with $A_{0}$ being the undeformed area of the specimen, $R_{i, 0}$ being the underformed internal radius, $A_{\text {star }}(t)$ being the area enclosed inside the deformed internal perimeter of the specimen (calculated via image analysis) and $A_{\text {star }, 0}$ being the value of the latter before at the start of the recovery (corresponding to the point in time evidenced in Figure 3).

\subsection{Star folding model}

A finite element model was setup in order to reproduce the experimental configuration shown in Figure 2 and described in the previous section. The cyclic symmetry and two symmetry planes were exploited to simulate only $1 / 24^{\text {th }}$ of the full specimen geometry and proper boundary conditions were added to model. As can be seen in Figure 4 the model mainly consists of a $2 \mathrm{D}$ analytic rigid part representing the punch and a $3 \mathrm{D}$ deformable part representing the specimen. Both the tangential symmetry planes are implemented in the model as rigid analytic surfaces to account for eventual self-contact interaction of the full specimen geometry. The mesh of the specimen consists of 2000 C3D20RHT (second order hexahedral elements with hybrid formulation and reduced integration) or 6000 C3D8RHT (first order hexahedral elements with hybrid formulation and reduced integration) depending on the 
thickness. Specifically, second order elements are used for thinner specimens to achieve a sufficient number of nodes along the thickness while maintaining a good element shape factor and a limited number of degrees of freedom.

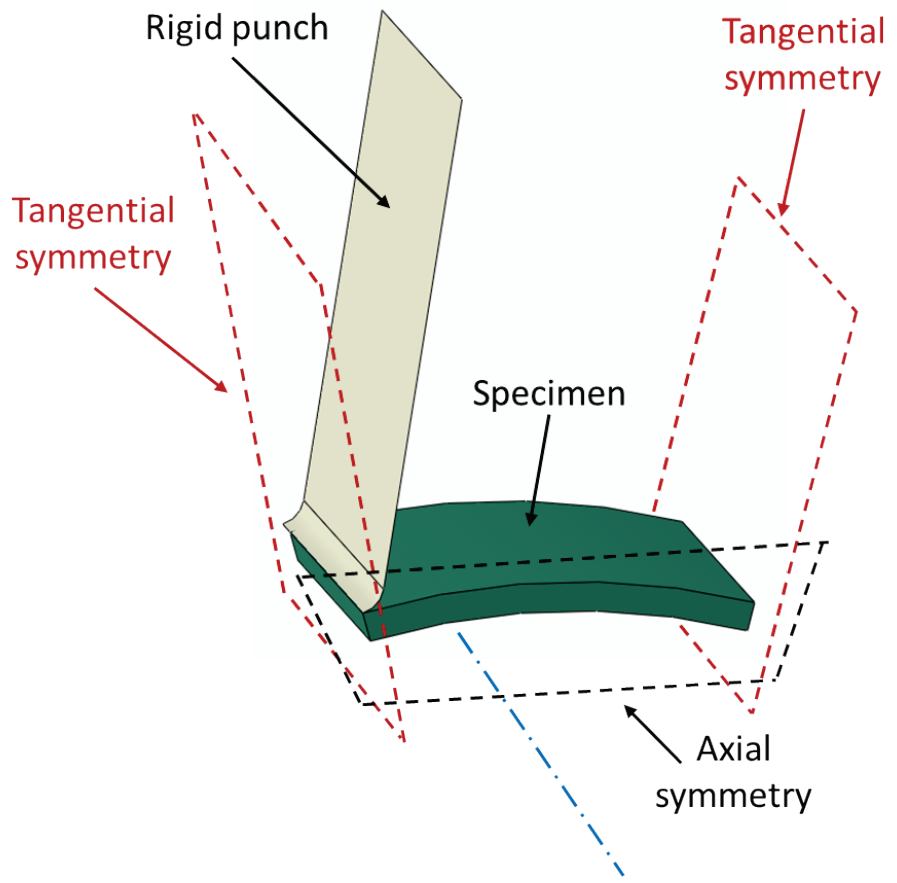

Figure 4: Finite element model assembly showing part geometries and boundary conditions.

The full thermal and strain histories follow the representative ones shown in Figure 3. Heat transfer phenomena were also simulated to catch the thermal history (and thus the shape recovery history) as correctly as possible by applying typical values for the free air convection coefficient and the epoxy specific heat.

The radial displacement applied to the punch and the isothermal temperature are applied according to Table 1 and this input data variety contributes in getting a wider validation spectrum. In the end, a video output of the simulation is used to calculate the inner area of the section through a custom image analysis algorithm developed from previous ones adopted for similar applications [28-30].

\section{RESULTS}

Results of the experimental material characterization and the finite element simulations are reported below.

\subsection{Material characterization}

Uniaxial compression data for three different samples in their rubbery state can be seen in Figure 5. A suitable data fit is achieved with a classical incompressible neo-Hookean strain energy $(W)$ function [31] as per eq. (6):

$$
W=C_{10}\left(I_{1}-3\right)
$$

with $I_{1}$ being the first invariant of the right Cauchy-Green deformation tensor and the neoHookean coefficient $C_{10}=0.277 \mathrm{MPa}$. 


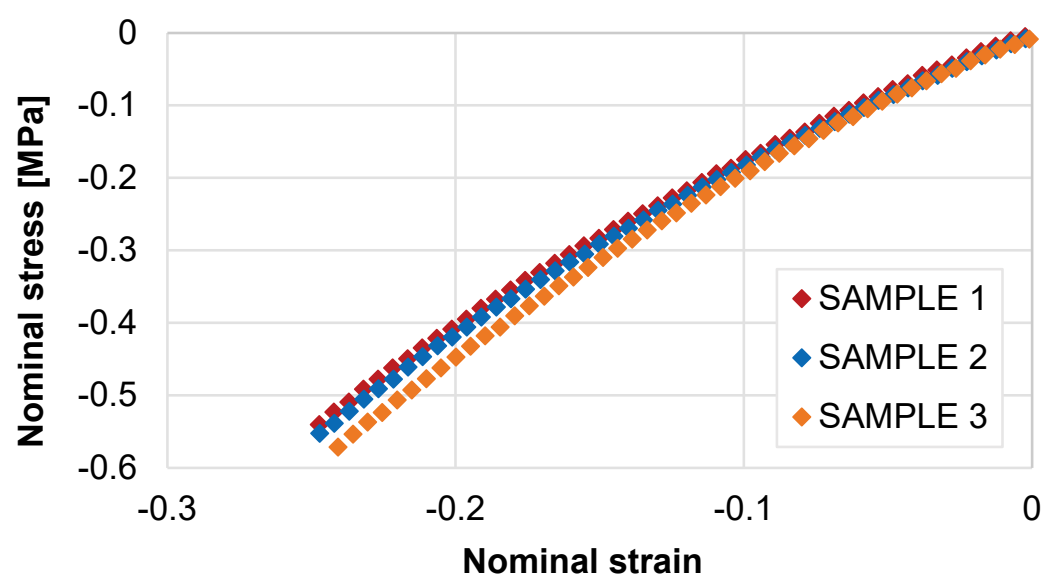

Figure 5: Uniaxial compression test data for three cylindrical epoxy samples in their rubbery state.

Raw data of a multifrequency DMA test are shown in Figure 6. The typical trend of the storage modulus for a viscoelastic polymer as function of frequency, and for various temperatures, can be appreciated: each curve represents an isothermal frequency sweep with the top and bottom curves being mostly overlapped due to a lower sensitivity to temperature changes, and the central ones being way more spaced. In fact, while the top and the bottom curves are respectively associated with the glassy and the rubbery plateau of a typical sigmoidal master curve, the most temperature-sensitive region is in between them and associated with the transition (around $\mathrm{T}_{\mathrm{g}}$ ) from one behavior to the other. Small additional relaxation effects, occurring at high temperature and/or at low frequencies, can also be seen at the right bottom of the figure.

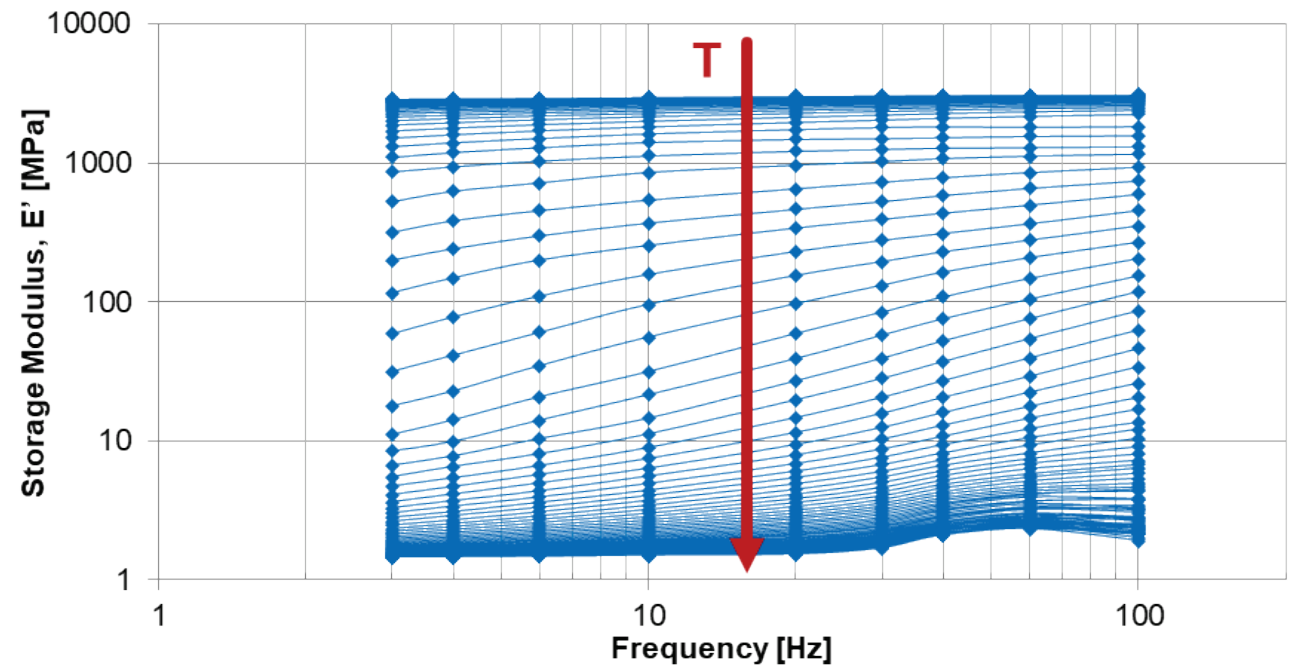

Figure 6: DMA data of the tensile storage modulus for a multifrequency test. Each curve corresponds to sweep from 3 to $100 \mathrm{~Hz}$ at a different temperature.

Shifting the curves along the frequency axis through an appropriate shift factor function $a_{T}(T)$ leads to the complete master curve as a function of the reduced frequency $f_{R}=a_{T} f$. The final storage modulus master curve can be appreciated in Figure 7 (a) as the black curve with the typical sigmoidal trend. Figure 7 also shows the optimization results in terms of storage shear modulus $G^{\prime}$, loss shear modulus $G^{\prime \prime}$ and dissipation factor, calculated as $G^{\prime \prime} / G^{\prime}, \tan (\delta)$. Again, all the trends are quite typical for a viscoelastic polymer. 

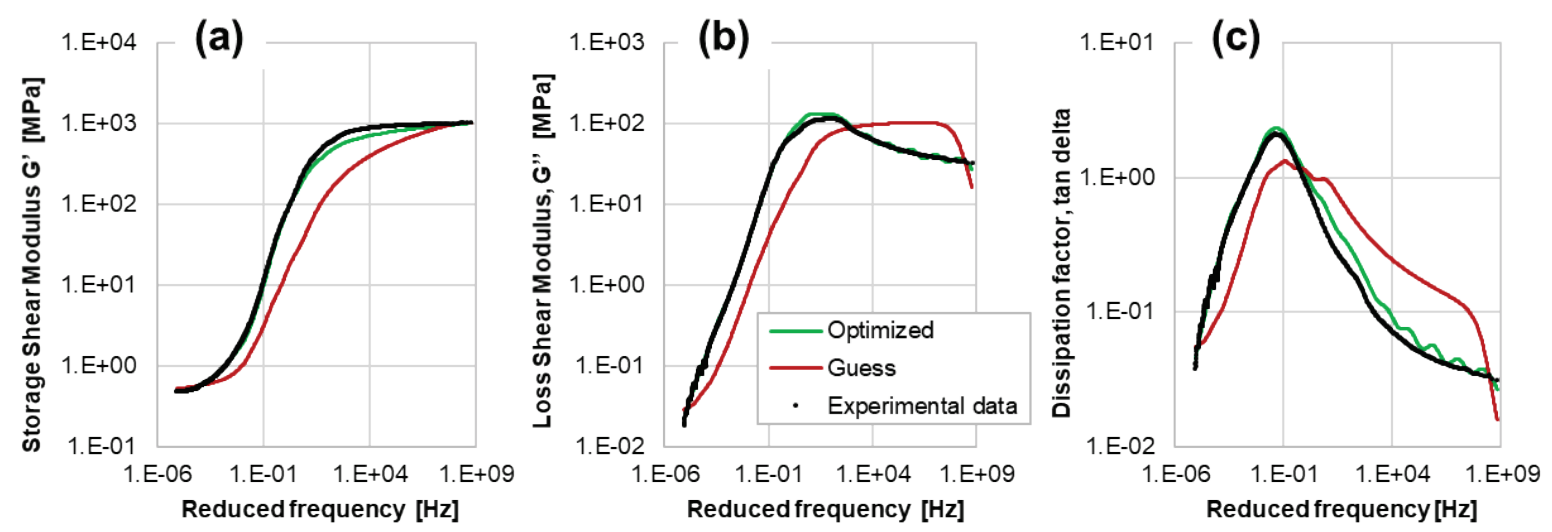

Figure 7: Results of the optimization procedure in terms of experimental against guess and optimized curves. Storage shear modulus, $G^{\prime}$, is shown in (a), loss shear modulus, $G^{\prime \prime}$, is reported in (b) and the dissipation factor $\left(G^{\prime \prime} / G^{\prime}\right)$, tan delta, is shown in (c). All curves refer to a temperature of $\approx 42.3^{\circ} \mathrm{C}$.

A very good agreement between experimental data and numerical fitting is shown for all DMA data along the whole reduced frequency range measured. While typical fluctuations due to the use of a Prony series representing the generalized Maxwell model are not particularly evident due to the high number of terms used, loss modulus and tan delta still show the fluctuations in the high reduced frequency region. However, the amplitude of these fluctuations being very small confirms the effectiveness of our optimization routine. Table 2 shows the values of the $26\left(\tau_{i}, g_{i}\right)$ pairs defining the Prony series used for the finite element simulations.

\begin{tabular}{|c|c|c|c|c|c|c|}
$i$ & $\tau_{i}$ & $g_{i}$ & $i$ & $\tau_{i}$ & $g_{i}$ \\
\hline 1 & $4.88 \mathrm{E}-10$ & $5.31 \mathrm{E}-02$ & & 14 & $1.40 \mathrm{E}-02$ & $5.15 \mathrm{E}-02$ \\
2 & $5.61 \mathrm{E}-09$ & $5.56 \mathrm{E}-02$ & & 15 & $2.62 \mathrm{E}-02$ & $4.23 \mathrm{E}-02$ \\
3 & $5.63 \mathrm{E}-08$ & $5.35 \mathrm{E}-02$ & & 16 & $5.08 \mathrm{E}-02$ & $3.63 \mathrm{E}-02$ \\
4 & $6.22 \mathrm{E}-07$ & $7.53 \mathrm{E}-02$ & & 17 & $6.09 \mathrm{E}-02$ & $3.32 \mathrm{E}-02$ \\
5 & $9.72 \mathrm{E}-06$ & $1.04 \mathrm{E}-01$ & & 18 & $2.14 \mathrm{E}-01$ & $4.70 \mathrm{E}-02$ \\
6 & $8.73 \mathrm{E}-05$ & $2.96 \mathrm{E}-02$ & & 19 & $5.57 \mathrm{E}-01$ & $2.08 \mathrm{E}-02$ \\
7 & $1.05 \mathrm{E}-04$ & $5.53 \mathrm{E}-02$ & & 20 & $2.57 \mathrm{E}+00$ & $4.52 \mathrm{E}-03$ \\
8 & $4.18 \mathrm{E}-04$ & $3.56 \mathrm{E}-02$ & & 21 & $5.83 \mathrm{E}+00$ & $9.67 \mathrm{E}-04$ \\
9 & $6.33 \mathrm{E}-04$ & $7.85 \mathrm{E}-02$ & & $3.91 \mathrm{E}+01$ & $7.22 \mathrm{E}-04$ \\
10 & $1.31 \mathrm{E}-03$ & $6.69 \mathrm{E}-02$ & & 23 & $2.85 \mathrm{E}+02$ & $3.60 \mathrm{E}-04$ \\
11 & $3.48 \mathrm{E}-03$ & $4.12 \mathrm{E}-02$ & $2.11 \mathrm{E}+03$ & $4.86 \mathrm{E}-05$ \\
12 & $3.61 \mathrm{E}-03$ & $5.35 \mathrm{E}-02$ & 26 & $1.14 \mathrm{E}+04$ & $1.18 \mathrm{E}-05$ \\
13 & $1.20 \mathrm{E}-02$ & $5.90 \mathrm{E}-02$ & & $1.14 \mathrm{E}+04$ & $3.37 \mathrm{E}-06$
\end{tabular}

Table 2: Prony parameters resulting from the optimization procedure on DMA data.

Figure 8 shows the trend of the shift factors $\left(a_{T}\right)$ with respect to the temperature and a good fit of experimental data is achieved with a WLF time-temperature correlation (see eq. (1)) at a reference temperature of $\approx 42.3{ }^{\circ} \mathrm{C}$, with $C_{1}=9.33$ and $C_{2}=35.2{ }^{\circ} \mathrm{C}$. 


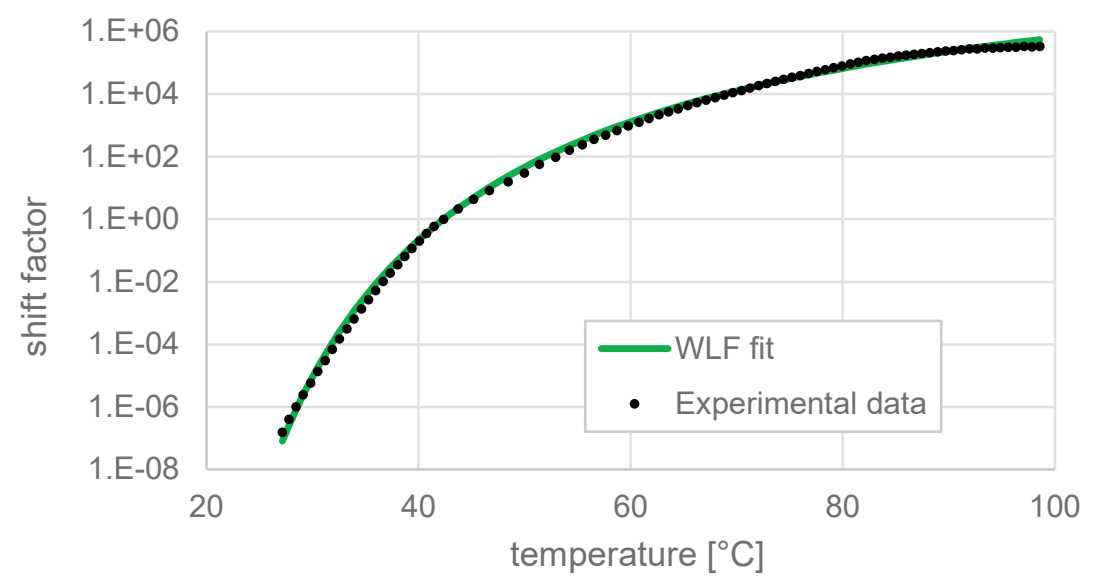

Figure 8: Shift factor $\left(a_{T}\right)$ as function of the temperature: experimental data and William-Landel-Ferry equation fit. Reference temperature is $\approx 42.3^{\circ} \mathrm{C}$.

\subsection{Star folding}

The time history of one specimen is shown in Figure 9 both in terms of experimental test and finite element simulation.

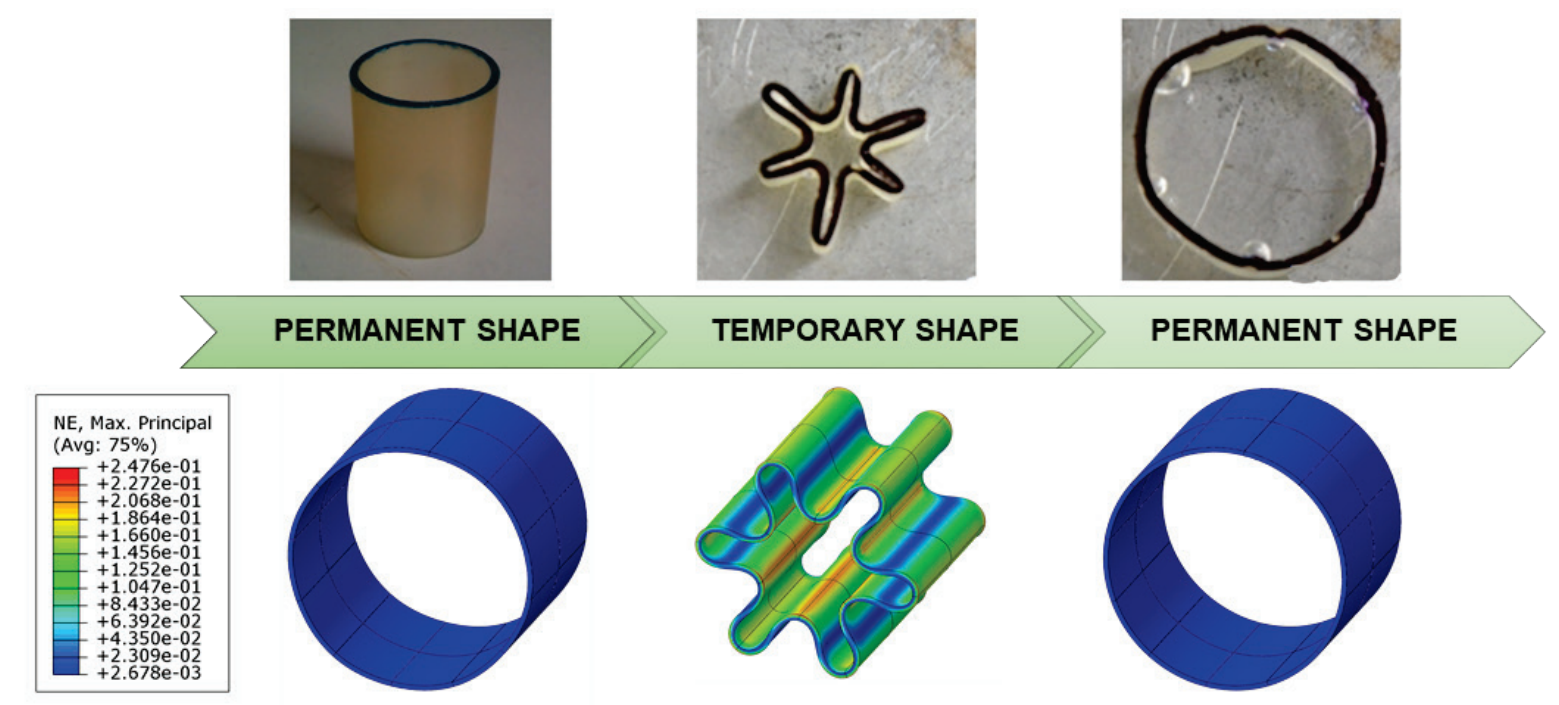

Figure 9: Representation of experimental and simulated deformation history. Maximum principal nominal strain is shown in the images from the finite element simulation.

As can be seen in Figure 10, the experimental data of the normalized deformation factor, $\varphi_{n}(t)$, are adequately approximated by the FEM isothermal recovery curves showing the effectiveness of our modelling approach. The results are particularly good when considering that experimental tests clearly show some non-ideal characteristics:

- Possible non-fully symmetric shapes (see Figure 9).

- Possible small heterogeneities in the resin composition.

- Incomplete shape recovery when the isothermal recovery is performed very close to $\mathrm{T}_{\mathrm{g}}$ (see curves at $37^{\circ} \mathrm{C}$ and $38.5^{\circ} \mathrm{C}$ in Figure 10 ).

In addition, all the FEM curves show good agreement with experimental data even though the isothermal recovery (especially near $\mathrm{T}_{\mathrm{g}}$ ) is the most critical shape recovery scenario and the 
tests were carried out at different temperatures, on different specimen thicknesses and at different strain levels.
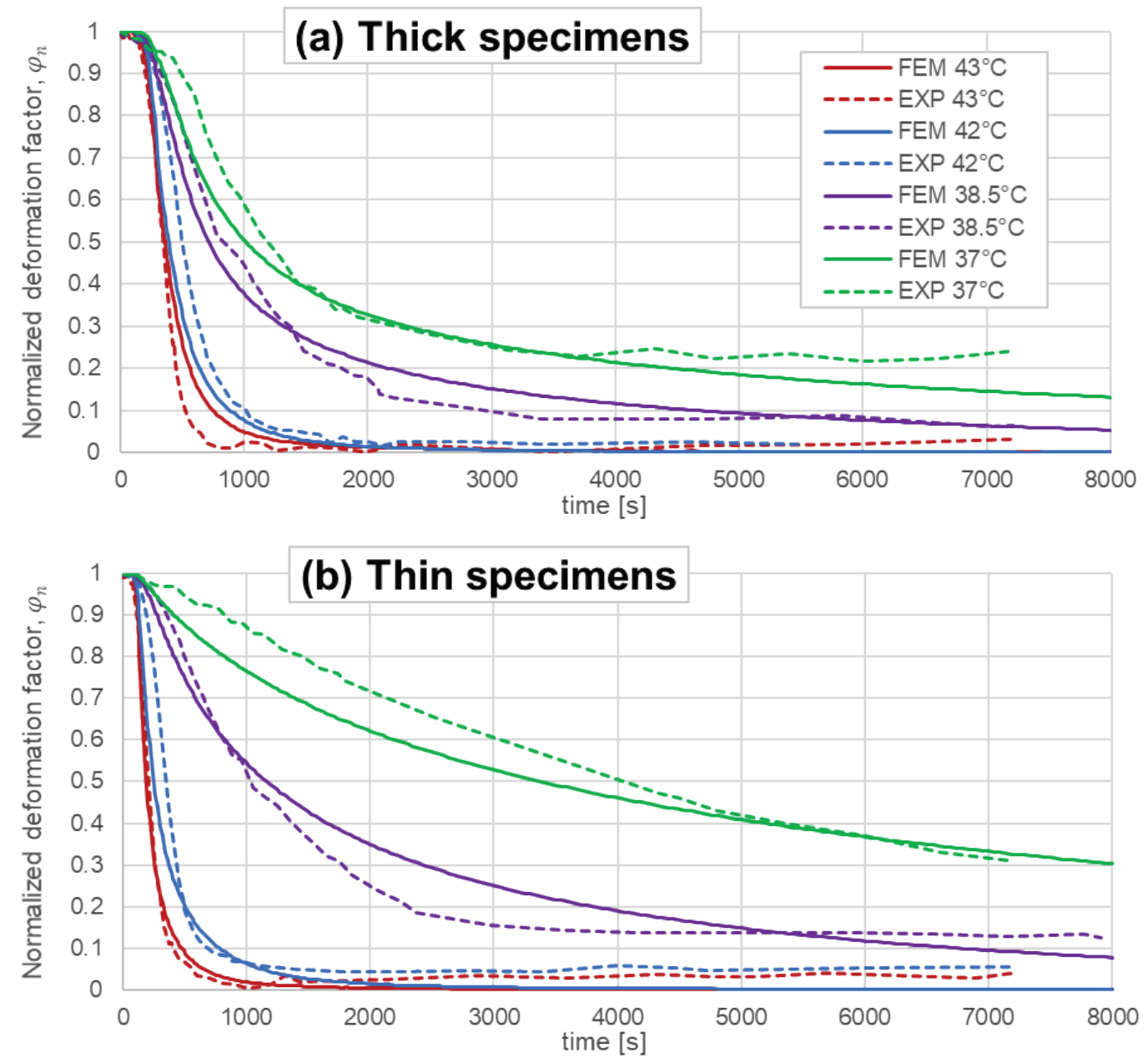

Figure 10: Normalized deformation factor $\varphi_{n}$ versus time for both the thick (a) and thin (b) specimens. Solid lines represent finite element values calculated from finite element simulation while dashed lines represent values calculated from experimental data. $\varphi_{n}=1$ means fully deformed, $\varphi_{n}=0$ means full recovery.

\section{CONCLUSIONS}

In this paper a feasible characterization and modellization for shape memory polymers is proposed. The proposed approach relies on standard uniaxial and DMA tests to gather all the necessary experimental data. While uniaxial test data are used to fit a hyperelastic strain energy which describes the time-independent material behavior, DMA data serve the purpose of describing time and temperature dependency. Specifically, a Prony series is used to describe a generalized Maxwell model together with a William-Landel-Ferry (WLF) equation to include a general time-temperature reduction scheme.

Neo-Hookean and WLF parameters are fit via standard non-linear optimization techniques, a custom optimization routine was coded to fit the Prony series parameters. This routine consists of a series of non-linear optimizers based on smart evolving guess values and constraints, which are arranged in three subsequent chains: while the first one performs a gross optimization, the second and the third ones perform the refinement. This fitting code guarantees a robust 
parameters optimization and consistent results while offering an easy-to-use GUI to load DMA data and to customize the main optimization settings.

A custom device was then used to deform and "program" eight circular tubular specimens to a six-pointed star shape at different strain levels. The shape recovery was then stimulated in isothermal conditions and the shape evolution was measured and compared to finite element simulations. Isothermal conditions near $T_{g}$ were specifically chosen due to slow recoveries being usually harder to predict and thus allowing for better considerations in terms of model validation. Finite element simulations results showed a good agreement for both the specimen thicknesses and all the temperature and strain ranges tested demonstrating the suitability of our testing, fitting and modelling methodologies.

\section{ACKNOWLEDGMENTS}

This is a pre-copyedited version of a contribution published in Carcaterra A., Paolone A., Graziani G. (eds) Proceedings of XXIV AIMETA Conference 2019. AIMETA 2019. Lecture Notes in Mechanical Engineering. Springer, Cham. The definitive authenticated version is available online via https://doi.org/10.1007/978-3-030-41057-5_56".

\section{REFERENCES}

[1] L. Sun et al., "Stimulus-responsive shape memory materials: A review," Materials \& Design, vol. 33, pp. 577-640, Jan. 2012.

[2] Y. Zhou and W. M. Huang, "Shape Memory Effect in Polymeric Materials: Mechanisms and Optimization," Procedia IUTAM, vol. 12, pp. 83-92, Jan. 2015.

[3] J. Leng, X. Lan, Y. Liu, and S. Du, "Shape-memory polymers and their composites: Stimulus methods and applications," Progress in Materials Science, vol. 56, no. 7, pp. 1077-1135, Sep. 2011.

[4] J. Hu, Y. Zhu, H. Huang, and J. Lu, "Recent advances in shape-memory polymers: Structure, mechanism, functionality, modeling and applications," Progress in Polymer Science, vol. 37, no. 12, pp. 1720-1763, Dec. 2012.

[5] S. Pandini, A. Avanzini, D. Battini, M. Berardi, F. Baldi, and F. Bignotti, "Shape memory behavior of epoxy-based model materials: Tailoring approaches and thermomechanical modeling," in AIP Conference Proceedings 1736, 2016, pp. 020175-1020175-4.

[6] T. Xie and I. A. Rousseau, "Facile tailoring of thermal transition temperatures of epoxy shape memory polymers," Polymer, vol. 50, no. 8, pp. 1852-1856, Apr. 2009.

[7] F. Xie, L. Huang, J. Leng, and Y. Liu, "Thermoset shape memory polymers and their composites," Journal of Intelligent Material Systems and Structures, vol. 27, no. 18, pp. 2433-2455, Nov. 2016.

[8] J. Karger-Kocsis and S. Kéki, "Review of progress in shape memory epoxies and their 
composites," Polymers, vol. 10, no. 1, pp. 1-38, 2017.

[9] K. S. Santhosh Kumar, R. Biju, and C. P. Reghunadhan Nair, "Progress in shape memory epoxy resins," Reactive and Functional Polymers, vol. 73, no. 2, pp. 421-430, Feb. 2013.

[10] F. Aggogeri, A. Avanzini, A. Borboni, and S. Pandini, "A Robot Gripper in Polymeric Material for Solid Micro-Meso Parts," International Journal of Automation Technology, vol. 11, no. 2, pp. 311-321, Mar. 2017.

[11] J. S. Arrieta, J. Diani, and P. Gilormini, "Experimental and modelling studies of the shape memory properties of amorphous polymer network composites," Smart Materials and Structures, vol. 23, no. 9, 2014.

[12] J. Diani, P. Gilormini, C. Frédy, and I. Rousseau, "Predicting thermal shape memory of crosslinked polymer networks from linear viscoelasticity," International Journal of Solids and Structures, vol. 49, no. 5, pp. 793-799, 2012.

[13] S. Arrieta, J. Diani, and P. Gilormini, "Experimental characterization and thermoviscoelastic modeling of strain and stress recoveries of an amorphous polymer network," Mechanics of Materials, vol. 68, pp. 95-103, 2014.

[14] Á. Kuki, K. Czifrák, J. Karger-Kocsis, M. Zsuga, and S. Kéki, “An approach to predict the shape-memory behavior of amorphous polymers from Dynamic Mechanical Analysis (DMA) data," Mechanics of Time-Dependent Materials, vol. 19, no. 1, pp. 8793, 2015.

[15] E. Ghobadi, R. Sivanesapillai, J. Musialak, and H. Steeb, “ Modeling Based Characterization of Thermorheological Properties of Polyurethane ESTANE TM ," International Journal of Polymer Science, vol. 2016, pp. 1-11, 2016.

[16] S. Pandini, F. Bignotti, F. Baldi, L. Sartore, G. Consolati, and G. Panzarasa, "Thermomechanical and large deformation behaviors of antiplasticized epoxy resins: Effect of material formulation and network architecture," Polymer Engineering \& Science, vol. 57, no. 6, pp. 553-565, Jun. 2017.

[17] R. M. Christensen, Theory of viscoelasticity : an introduction. Academic Press, Elsevier, 1982.

[18] J. Bergström and J. Bergström, "Linear Viscoelasticity," Mechanics of Solid Polymers, pp. 309-351, Jan. 2015.

[19] W. N. Findley, J. S. Lai, and K. Onaran, Creep and relaxation of nonlinear viscoelastic materials, with an introduction to linear viscoelasticity. North-Holland Pub. Co., 1976.

[20] S. W. Park and R. A. Schapery, "Methods of interconversion between linear viscoelastic material functions. Part I- a numerical method based on Prony series," International Journal of Solids and Structures, vol. 36, no. 11, pp. 1653-1675, Apr. 1999.

[21] T. O'Haver, A pragmatic introduction to signal processing with applications in scientific measurement, June 2019. ONLINE @ https://terpconnect.umd.edu/ toh/spectrum, 2019.

[22] D. S. G. Pollock, Handbook of Time Series Analysis, Signal Processing, and Dynamics. Elsevier, 1999. 
[23] L. M. Rios and N. V. Sahinidis, "Derivative-free optimization: A review of algorithms and comparison of software implementations," Journal of Global Optimization, vol. 56, no. 3, pp. 1247-1293, 2013.

[24] S. Das and P. N. Suganthan, "Differential evolution: A survey of the state-of-the-art," IEEE Transactions on Evolutionary Computation, vol. 15, no. 1, pp. 4-31, 2011.

[25] W. M. Huang, B. Yang, N. Liu, and S. J. Phee, "Water-responsive programmable shape memory polymer devices," International Conference on Smart Materials and Nanotechnology in Engineering, vol. 6423, no. November 2007, p. 64231S, 2007.

[26] C. Azra, C. J. G. Plummer, and J.-A. E. Månson, "Isothermal recovery rates in shape memory polyurethanes," Smart Materials and Structures, vol. 20, no. 8, p. 082002, Aug. 2011.

[27] C. Azra, C. J. G. Plummer, and J.-A. E. Månson, "Dynamic mechanical analysis for rapid assessment of the time-dependent recovery behavior of shape memory polymers," Smart Materials and Structures, vol. 22, no. 7, p. 075037, Jul. 2013.

[28] A. Avanzini and D. Battini, "Structural analysis of a stented pericardial heart valve with leaflets mounted externally," Proceedings of the Institution of Mechanical Engineers, Part H: Journal of Engineering in Medicine, vol. 228, no. 10, pp. 985-995, 2014.

[29] A. Avanzini and D. Battini, "FEM Simulation of Subintimal Angioplasty for the Treatment of Chronic Total Occlusions," Mathematical Problems in Engineering, pp. 113, 2018.

[30] A. Avanzini and D. Battini, "Integrated experimental and numerical comparison of different approaches for planar biaxial testing of a hyperelastic material," Advances in Materials Science and Engineering, vol. 2016, pp. 1-12, 2016.

[31] R. S. Rivlin, "Large Elastic Deformations of isotropic materials. IV. Further developments of the general theory," Philosophical Transactions of the Royal Society A: Mathematical, Physical and Engineering Sciences, vol. 241, no. October, pp. 379-397, Oct. 1948. 